\title{
The Emergency Rooms and Emergency Medicine should not be the answer to the weakness of the Italian National Health System
}

\author{
Erika Poggiali, ${ }^{1}$ Greta Barbieri, ${ }^{2,3}$ Veronica Salvatore, ${ }^{4}$ Francesco Salinaro ${ }^{5}$ \\ ${ }^{1}$ Emergency Department, Guglielmo da Saliceto Hospital, Piacenza; ${ }^{2}$ Department of Surgical, Medical, \\ Molecular and Critical Area Pathology, University of Pisa, Pisa; ${ }^{3}$ Emergency Medicine Department, Pisa \\ University Hospital, Pisa; ${ }^{4}$ Emergency Department, Medicina d'Urgenza e Pronto Soccorso, IRCCS Azienda \\ Ospedaliero-Universitaria di Bologna, Bologna; 5Emergency Medicine Unit, IRCCS Polyclinic San Matteo \\ Foundation, Department of Internal Medicine, University of Pavia, Pavia, Italy
}

\section{Dear Editors,}

The COVID-19 pandemic has highlighted several and wellknown limits of the Italian national health system, including the weakness of the territorial medical services and the absence of dedicated structures for patients affected by chronic or end-stage diseases in need of long hospitalization, or with social difficulties. It is clear that the Emergency Department (ED) cannot be the answer to these deeply rooted problems, but we are aware that, in the absence of a prompt and effective reorganization of the health system by the Italian government, the Emergency Room (ER) will

Correspondence: Erika Poggiali, Emergency Department, Guglielmo da Saliceto Hospital, Via Giuseppe Taverna 49, 29121 Piacenza, Italy. Tel.: +39.0523.303044

E-mail: poggiali.erika@gmail.com

Key words: Emergency room; emergency medicine; national health system; health crisis; COVID-19.

Contributions: All the authors equally contributed to the manuscript, approved the final version and stated the integrity of the whole work.

Conflicts of interest: The authors declare no conflict of interest. EP, GB, VS and FS are members of the editorial board of Emergency Care Journal.

Availability of data and materials: All data are fully available upon reasonable request from Erika Poggiali, poggiali.erika@gmail.com

Ethics approval and consent to participate: Not applicable.

Informed consent: Not applicable.

Acknowledgements: The authors thank the Emergency Department of their hospitals for the precious help, strength, complete dedication and resiliency every time and everywhere.

Received for publication: 29 November 2021.

Revision received: 29 November 2021.

Accepted for publication: 29 November 2021.

This work is licensed under a Creative Commons Attribution 4.0 License (by-nc 4.0).

${ }^{\circ}$ Copyright: the Author(s), 2021

Licensee PAGEPress, Italy

Emergency Care Journal 2021; 17:10304

doi:10.4081/ecj.2021.10304 often remain the only place where patients can look for a response to their health problems, whether or not they represent a real emergency. Over the last decades, the role of EDs has necessarily changed in response to the weaknesses of the national health system and to the lack of an efficient territorial network. In fact, nowadays EDs provide not only acute emergency services, but also primary health care and social support to people in need. These non-institutional activities require great amounts of energy and resources. As a consequence, ERs are often overcrowded, and their patients must face long waiting times to be visited and even longer boarding times to be hospitalized. More importantly, the management of real emergencies can be endangered in this context, since concentration, energy and collaboration between medical and nursing staff, which are crucial for a positive outcome, are themselves at danger. The step is short to the inevitable cracking of the relationship between patients and clinicians, to the loss of mutual trust and respect, to growth of discomfort and frustration, to the increase in episodes of violence, and of burn-out phenomena. All this is what pushes emergency doctors to abandon the EDs and the emergency medicine specialty.

In 2019, before the COVID-19 pandemic, 24 million patients (one third of the population) were admitted to the Italian EDs at a rate of one every 90 seconds. But, looking at the other side of the coin, we know that EDs faced these numbers lacking at least 4000 emergency physicians and 10000 nurses. These numbers are impressive, but not new to the Italian government. Changes are necessary to avoid the complete collapse of the emergency services and, as a consequence, of the entire national health system. Strong initiatives should be taken right now. Recently, the Italian Society of Emergency Medicine (SIMEU) has organized a sit-in in Rome with the end goal to quickly work towards a solution with the Italian health minister, Roberto Speranza. The crisis of the emergency medicine is the reflection of a deeper and hidden crisis of the Italian national health system. The pandemic has landed in an emergency medical system already lacerated by deep difficulties due to the lack of a well-structured and organized territorial medical service and to the absence of long-stay hospitals. The solution cannot and must not be looked for within the EDs, but rather in the development of a serious project for the complete reorganization of the national health system. Within this framework, if we want to avoid a crisis with no escape, the EDs should be valued as crucial hubs for patients' acute care pathways, and should be finely interconnected with the whole territory and the other hospital departments.

We want to remember that emergency medicine is the medical specialty concerned with the care of illnesses or injuries requiring immediate medical attention. Emergency physicians or "ER doctors" continuously study to care for unscheduled and undifferenti- 
ated patients of all ages with the primary mission to save lives and respond to acute and severe illness. They have great responsibilities in life-threatening conditions, dealing with which they need to work as part of a team, and must be able to think and act quickly, to work out a differential diagnosis and to follow the appropriate course of treatment. All this needs a good organization and the best working conditions, that currently cannot be guaranteed because of the overcrowding of the ERs and the limited human and space resources.
Those who write are four young emergency doctors who believe that their work is the most beautiful in the world, a useful and worthwhile one, even when tiring or difficult, or even painful at times. We all strongly believe that "emergency medicine is the most interesting 15 minutes of every other specialty" (Dan Sandberg, BEEM Conference, 2014) and we bravely hope that the future of the Italian emergency medicine will be better than now. 